\title{
A Rare Case of Bilateral Pheochromocytoma
}

\author{
Senthil K Azhisoor Chandrashekhar ${ }^{1}$, Pallavi B Kothe ${ }^{2 \odot}$, Muthuvel G Esakki ${ }^{3}$, Rajalakshmi K Venkatraju ${ }^{4}$, Priya D Venugopalan ${ }^{5}$
}

\begin{abstract}
Pheochromocytoma is a rare adrenal gland tumor, which can present bilaterally but not all cases have indications of bilateral adrenalectomy. Hereby presenting a case of a young girl of bilateral adrenal pheochromocytoma for which bilateral adrenalectomy (right side cortical sparing) was done. Correct diagnosis and timely intervention are important to prevent lifelong complications.

Keywords: 131 I MIBG scan, Bilateral Pheochromocytoma, Bilateral adrenalectomy, Cortical-sparing adrenalectomy, Hypertension. World Journal of Endocrine Surgery (2021): 10.5005/jp-journals-10002-1400
\end{abstract}

\section{BACKGROUND}

Pheochromocytoma is a neuroectodermal tumor of the catecholamine-producing chromaffin cells of the adrenal medulla. Hereditary pheochromocytomas occur at a younger age and tend to be multifocal and/or bilateral at presentation. Metaiodobenzylguanidine (MIBG) scintigraphy, urinary or serum metanephrines (normetanephrine and metanephrine), has been used to evaluate patients with pheochromocytoma. Pheochromocytoma is a surgical disease. Complete resection of the tumor is advised whenever possible. Hereby discussing a case of bilateral adrenal incidentaloma in young 19-year-old female presenting with hypertension and cerebral vascular episode where further evaluation revealed the diagnosis of bilateral pheochromocytoma and bilateral adrenalectomy (right-side cortical sparing) was done without significant perioperative complications.

\section{Case Description}

A 19-year-old female presented with complaint of an episode of spontaneous onset of seizure with jerky movements of hands and legs with no uprolling of eyes. It was associated with hypertension. There was no history of trauma, headache, blurring of vision, nausea, and vomiting.

Patient was evaluated and $\mathrm{MRI}, \mathrm{CT}$ brain revealed acute intraparenchymal hemorrhagic foci in right gangliocapsular region with mass effect in form of compression in ipsilateral lateral ventricle. Cerebral angiogram was followed, which revealed $35 \times 16 \mathrm{~mm}$ with density of 57-71 HU intracapsular hemorrhage in right lentiform nucleus and external capsule, perifocal edema, and midline shift of $3 \mathrm{~mm}$. She was started on conservative management of LEVETIRACETAM, PHENYTOIN, MANNITOL, and $3 \% \mathrm{NaCl}$. Her repeat $\mathrm{CT}$ brain images did not show any significant interval changes. There was no remnant neurofocal deficit or any repeat episodes of seizures and patient was symptomatically better and continued on tablet LEVETIRACETAM.

Her persistently high BP warranted the necessity of antihypertensives where she was started with tablet AMLODIPINE, tablet TELMISARTAN, tablet NICARDIA-R, tablet MET-XL, whose doses were adjusted according to her charts. To evaluate the cause of her hypertension, renal Doppler was done which revealed high PSV in right renal artery, left renal artery, and left hilar artery, which suggested an obstructive pathology. Renal angiogram was undertaken which revealed bilateral adrenal gland lesion causing
${ }^{1}$ Department of Surgical Oncology, Saveetha Medical College and Hospital, Chennai, Tamil Nadu, India

${ }^{2}$ Department of General Surgery, Saveetha Medical College and Hospital, Thandalam, Chennai, Tamil Nadu, India

${ }^{3}$ Department of Nephropathology, Saveetha Medical College and Hospital, Saveetha University, Chennai, Tamil Nadu, India

${ }^{4}$ Institute of Non-Communicable Diseases, Government Royapettah Hospital \& Government Kilpauk Medical College, Chennai, Tamil Nadu, India

${ }^{5}$ Government Medical College, Omandurar Government Estate, Chennai, Tamil Nadu, India

Corresponding Author: Pallavi B Kothe, Department of General Surgery, Saveetha Medical College and Hospital, Thandalam, Chennai, Tamil Nadu, India, Phone: +91 9960032598, e-mail: pallavi_kothe111@rediffmail.com

How to cite this article: Azhisoor Chandrashekhar SK, Kothe PB, Esakki MG, et al. A Rare Case of Bilateral Pheochromocytoma. World J Endoc Surg 2021;13(2):57-60.

Source of support: Nil

Conflict of interest: None

mass effect highly suspicious of pheochromocytoma. Right adrenal gland measured $2.3 \times 1.8 \times 2.0 \mathrm{~cm}$ abutting adjacent structures. Left adrenal gland lesion measured $5.2 \times 3.2 \times 4.5 \mathrm{~cm}$ with multiple calcific foci and non-enhancing areas causing mass effect (Figs 1 and 2).

On further biochemical evaluation, her 24 hours urine metanephrine was $55.2(<350)$, VMA was $8.2(<13.6)$, C3 was 2.13 (0.9-1.8), C4 was $0.38(0.1-0.4)$, plasma metanephrine was $103(<65)$, and plasma normetanephrine was $554.71(<196)$. Her thyroid profile and other parameters were within normal range.

Further evaluation was done with 131I MIBG scan, which showed concentrating tumor in bilateral adrenal gland suggestive of bilateral pheochromocytoma and no other paraganglioma (Figs 3 and 4).

As the diagnosis of pheochromocytoma was established, antihypertensive management was modified to include alpha blockers hence patient was started on tablet PHENOXYBENZAMINE $10 \mathrm{mg}$ BD along with tablet METXL $25 \mathrm{mg}$ BD and tablet NICARDIA-R $20 \mathrm{mg} \mathrm{BD}$ which she continued for 1 month till the day of surgery. Her blood pressure was vigilantly monitored and barring few hypertensive episodes per day was fairly under control and patient was asymptomatic. 


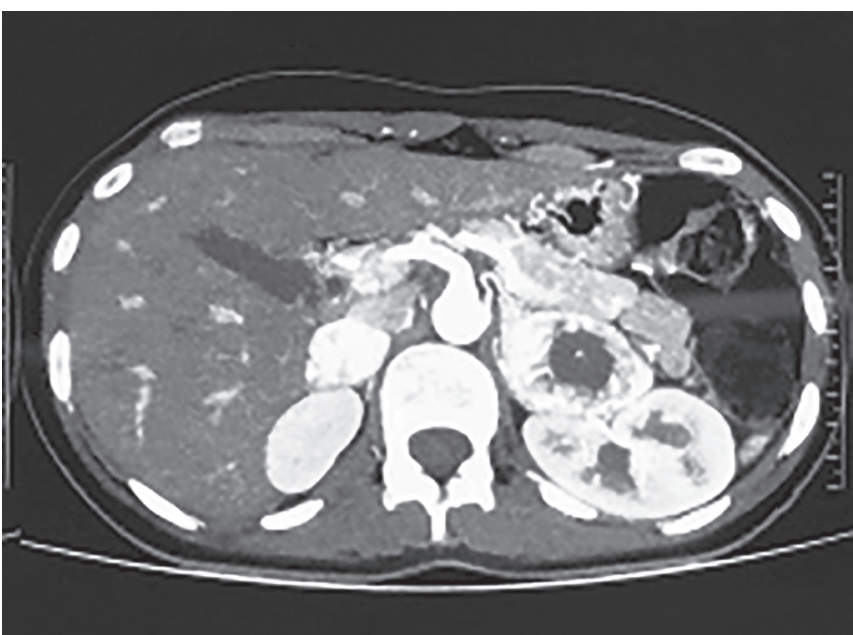

Fig. 1: Renal CT sagittal section, right adrenal gland measured $2.3 \times 1.8 \times 2.0 \mathrm{~cm}$ abutting adjacent structures such as IVC, segment $\mathrm{V}$ of liver, right crus of diaphragm and ipsilateral kidney. Left adrenal gland lesion measured $5.2 \times 3.2 \times 4.5 \mathrm{~cm}$ with multiple calcific foci and non-enhancing areas causing mass effect on stomach, splenic vein, body of pancreas, left crus of diaphragm, ipsilateral kidney, and renal vessel

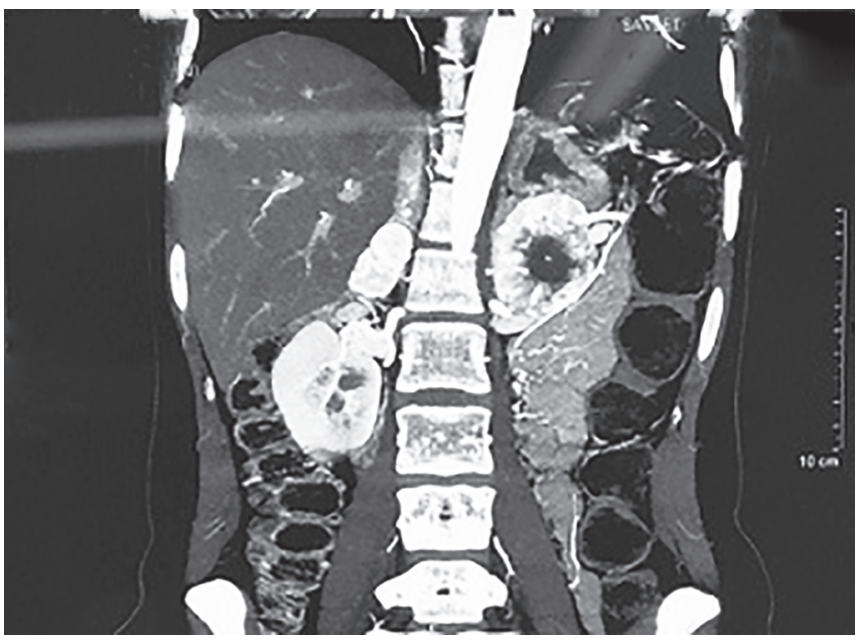

Fig. 2: Coronal section renal CT right adrenal gland measured $2.3 \times 1.8 \times 2.0 \mathrm{~cm}$ abutting adjacent structures such as IVC, segment $\mathrm{V}$ of liver, right crus of diaphragm, and ipsilateral kidney. Left adrenal gland lesion measured $5.2 \times 3.2 \times 4.5 \mathrm{~cm}$ with multiple calcific foci and non-enhancing areas causing mass effect on stomach, splenic vein, body of pancreas, left crus of diaphragm, ipsilateral kidney, and renal vessel

Preoperatively, necessary IV hydration and antiepileptics were given and surgery for bilateral adrenalectomy was planned. Under necessary anesthesia cover, with rooftop incision, right adrenal gland $(3 \times 3 \mathrm{~cm})$ was visualized after kocherization and mobilization of colon. Adrenal vein and artery were ligated and divided. Right adrenalectomy was uneventful without any intraoperative fluctuation of BP. To approach left adrenal gland tumor $(4.8 \times 4.5 \mathrm{~cm})$, pancreas and spleen were mobilized and multiple feeding vessels were ligated. Left adrenal vein was isolated, ligated, and divided. Left adrenalectomy and removal of specimen were involved with high intraoperative BP of 200/120, which was managed efficiently by anesthesia team. No other metastatic features were noted in intraabdominal examination of liver or nodes. Surgery was uneventful and patient's perioperative recovery was satisfactory (Fig. 5).

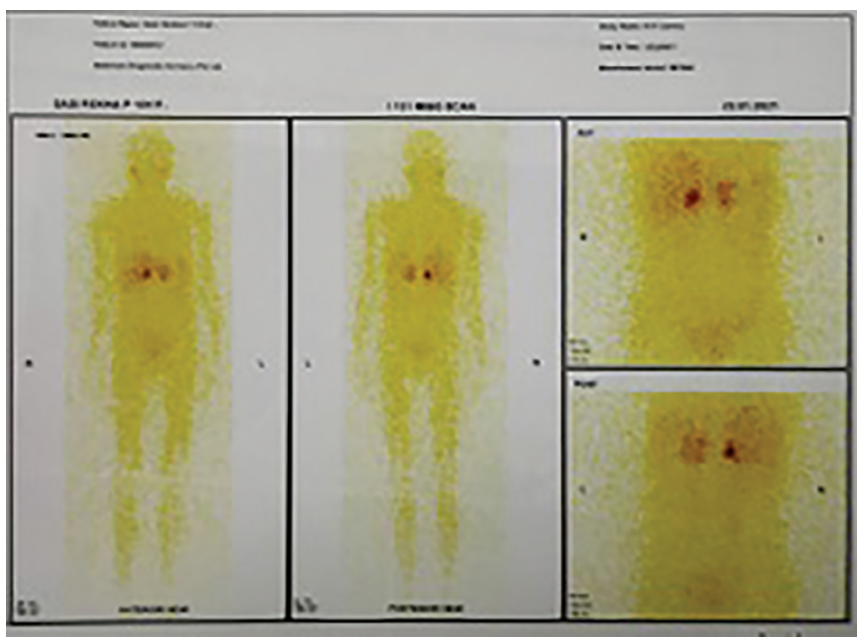

Fig. 3: Maximum intensity projection in MIBG
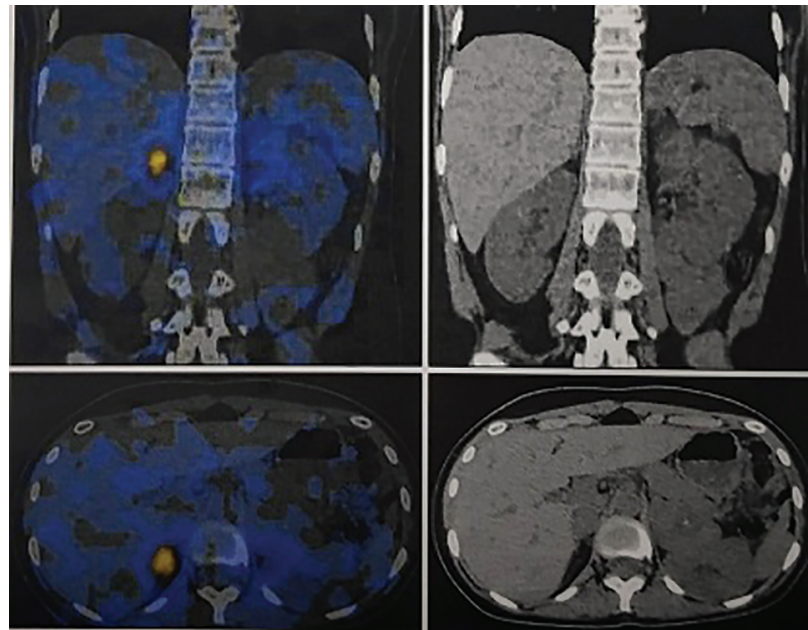

Fig. 4: Coronal section in MIBG

She was started on tablet fludrocortisone $0.1 \mathrm{mg}$, which was gradually tapered and stopped till POD 7. Initially IV HYDROCORTISONE and later oral was continued for the patient. She continued to get potassium correction in view of hypokalemia, which was gradually stopped and low salt IV albumin correction was given in view of hypoalbuminemia. Patient continued to take antiepileptic treatment.

Histopathology examination of bilateral adrenal gland confirmed bilateral pheochromocytoma showing fairly defined uniform nuclei and abundant clear cytoplasm arranged in Zellballen pattern with areas of hemorrhage and necrosis. Left adrenal mass showed focal areas of nuclear polymorphism (Fig. 6).

One month after the surgery, plasma metanephrine was $28.60 \mathrm{pg} / \mathrm{mL}$ and plasma normetanephrine was $110.7 \mathrm{pg} / \mathrm{mL}$. Patient was in regular follow-up and showed satisfactory recovery.

\section{Discussion}

Pheochromocytoma is a neuroectodermal tumor of the catecholamine-producing chromaffin cells of the adrenal medulla. Classically, pheochromocytoma has been called the "10\% tumor": $10 \%$ extra-adrenal, $10 \%$ familial, $10 \%$ bilateral, $10 \%$ pediatric, and $10 \%$ malignant. Nonhereditary cases of pheochromocytoma are most often diagnosed in the fourth and fifth decades of life. Familial tumors 


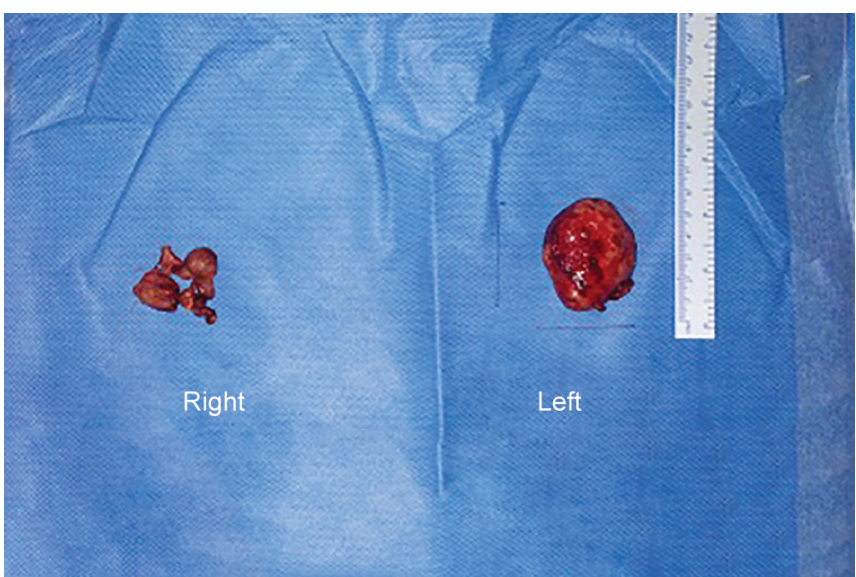

Fig. 5: Postoperative image of specimen of bilateral adrenal gland

present at a younger age. A hereditary syndrome is suspected when patients are found to have a pheochromocytoma at a young age, bilateral or recurrent disease, or extra-adrenal disease.

Paraganglioma (PGL) is an extra-adrenal tumor of the autonomic nervous system. When discovered during imaging accidentally, adrenal tumors are referred to as adrenal incidentalomas. Many syndromes are associated with pheochromocytoma such as neurofibromatosis type I with highest malignant potential, Von Hippel-Lindau Syndrome, multiple endocrine neoplasia. ${ }^{2}$

Paroxysmal hypertension is the classic presenting sign. Classical hallmark of pheochromocytoma is the triad of headache, episodic sudden perspiration, and tachycardia. More than $20 \%$ of patients can be asymptomatic.

The diagnosis of pheochromocytoma is made by excess secretion of catecholamines. Testing for urinary or serum metanephrines (normetanephrine and metanephrine) is done and represents the modern advancement in biochemical investigations. ${ }^{3,4}$

Imaging studies include MRI (the most sensitive modality), CT imaging, and 131I metaiodobenzylguanidine (MIBG) scanning. MIBG is a small-molecule analog of norepinephrine. With MIBG tagged with either 131I or 123l, MIBG scintigraphy has been used to evaluate patients with pheochromocytoma. FDG PET was equally effective, but was more sensitive in detecting metastatic disease. ${ }^{5}$

The main focus of preoperative preparation is adequate alpha-adrenergic blockade and restoration of fluid and electrolyte balance. Phenoxybenzamine is the most common a-blocker used for preoperative catecholamine blockade of pheochromocytoma. Careful preoperative preparation is required to prevent a cardiovascular crisis during surgery caused by excess catecholamine secretion. ${ }^{6,7}$

Pheochromocytoma is a surgical disease. Complete resection of the tumor is advised. Debulking and metastasectomy can be considered in patients who have significant resectable tumor slowly progressive disease presentation. ${ }^{1}$ A minimally invasive approach $^{8}$ is advised for patients with MEN II or von HippelLindau disease. The disease should be small, unilateral, and bilateral pheochromocytoma less than approximately $6 \mathrm{~cm}$ in size for minimal invasive surgery. ${ }^{9}$ In high-risk bilateral disease, partial cortical-sparing adrenalectomy is advised. Cortical-sparing adrenalectomy, either open or laparoscopic, ${ }^{8}$ has been performed successfully in patients with MEN $1{ }^{10}$ or von Hippel-Lindau disease with bilateral pheochromocytomas. This avoids lifelong steroid hormone replacement and Addisonian crisis risk.

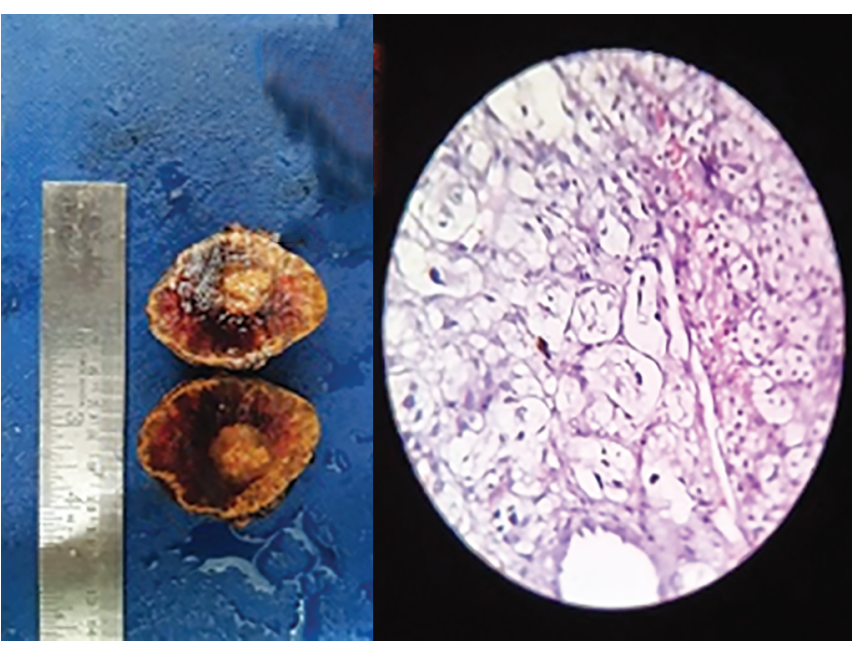

Fig. 6: Gross pathological cut section of left adrenal gland showing areas of necrosis, microscopicfairly defined uniform nuclei and abundant clear cytoplasm arranged in Zellballen pattern with adjacent normal tissue

Patients improve after surgical resection of pheochromocytoma. Hypertension may persist postoperatively in $25 \%$ of patients, especially in those with sustained hypertension preoperatively. Surgical resection has excellent prognosis. Disease may recur after many years of resection in approximately $16 \%$ cases. Hence, vigilant lifelong follow-up like measurement of plasma-free metanephrines at 1,6 , and 12 months postoperatively, ${ }^{3,4}$ then annually is advocated. Additionally cross-sectional imaging is also contributory.

The most common sites of metastasis are to bones, lungs, liver, and lymph nodes. Radiofrequency ablation (RFA) and transarterial chemoembolization (TACE) are other options for unresectable metastases. In clinically stable but poor surgical candidates,131I-MIBG can be used for locoregional control. ${ }^{11}$ 26,132 Cyclophosphamide-dacarbazine-based cytotoxic chemotherapy regimens are used in unresectable disease where other treatment options are exhausted.

\section{Conclusion}

Pheochromocytoma should be ruled out in young age uncontrolled hypertension. It is a surgically resectable disease which, if managed early, can improve the outcome of the surgery and quality of life of patient both systemically and hormonally. Team of specialists is imperative in managing the patient in perioperative time, which is an extremely high-risk area for both morbidity and mortality of patient in view of fluctuations of blood pressure and high risk of cardiovascular and cerebrovascular episodes. Postoperatively, patient needs to be managed by expert endocrinologist for lifelong hormonal supplementation. Regular follow-up with radiological investigations and oncological management are needed to ensure disease-free life of patient.

\section{Acknowledgment}

No funding was provided.

\section{OrCID}

Pallavi Baban Kothe ๑ http://orcid.org/0000-0002-6048-1518 


\section{References}

1. Giford RW, Jr, Kvale WF, Maher FT, et al. Clinical features, diagnosis and treatment of pheochromocytoma: A review of 76 cases. Mayo Clin Proc 1964;39:281-302.

2. Neumann HPH, Bausch B, McWhinney SR, et al. Germ-line mutations in nonsyndromic pheochromocytoma. N Engl J Med 2002;346: 1459-1466. DOI: 10.1056/NEJMoa020152

3. Sawka AM, Prebtani AP, Tabane $L$, et al. A systematic review of the literature examining the diagnostic efficacy of measurement of fractionated plasma free metanephrines in the biochemical diagnosis of pheochromocytoma. BMC Endocr Disord 2004;4:2. DOI: 10.1186/1472-6823-4-2a

4. Perry CG, Sawka AM, Singh R, et al. The diagnostic efficacy of urinary fractionated metanephrines measured by tandem mass spectrometry in detection of pheochromocytoma. Clin Endocrinol (Oxf) 2007;66:703-708. DOI: 10.1111/j.1365-2265.2007. 02805.x

5. Timmers HJ, Chen CC, Carrasquillo JA, et al. Comparison of ${ }^{18} \mathrm{~F}$-fuoroL-DOPA, ${ }^{18} \mathrm{~F}$-fuoro-deoxyglucose, and ${ }^{18} \mathrm{~F}$-fuorodopamine PET and ${ }^{123}$ I-MIBG scintigraphy in the localization of pheochromocytoma and paraganglioma. J Clin Endocrinol Metab 200994:4757-4767. DOI: $10.1210 /$ jc.2009-1248
6. Kinney MAO, Narr BJ, Warner MA. Perioperative management of pheochromocytoma. J Cardiothorac Vasc Anesth 2002;16:359-369. DOI: 10.1053/jcan.2002.124150

7. Brunaud L, Boutami M, Nguyen-Thi PL, et al. Both preoperative alpha and calcium channel blockade impact intraoperative hemodynamic stability similarly in the management of pheochromocytoma. Surgery 2014;156(6):1410-1417. DOI: 10.1016/j.surg.2014.08.022

8. Shen WT, Grogan R, Vriens M, et al. One hundred two patients with pheochromocytoma treated at a single institution since the introduction of laparoscopic adrenalectomy. Arch Surg 2010;145: 893-897. DOI: 10.1001/archsurg.2010.159

9. Shen WT, Sturgeon C, Clark OH, et al. Should pheochromocytoma size influence surgical approach? A comparison of 90 malignant and 60 benign pheochromocytomas. Surgery 2004;136(6):1129-1137. DOI: $10.1016 /$ j.surg.2004.05.058

10. Anouk S. Unilateral subtotal adrenalectomy for pheochromocytoma in multiple endocrine neoplasia type 2 patients: a feasible surgical strategy. Ann Surg 2011;254(6):1022-1027. DOI: 10.1097/ SLA.0b013e318237480c

11. Gonias S, Goldsby R, Matthay KK, et al. Phase II study of high-dose ${ }^{[1311]}$ metaiodobenzylguanidine therapy for patients with metastatic pheochromocytoma and paraganglioma. J Clin Oncol 2009;27: 4162-4168. DOI: 10.1200/JCO.2008.21.3496 